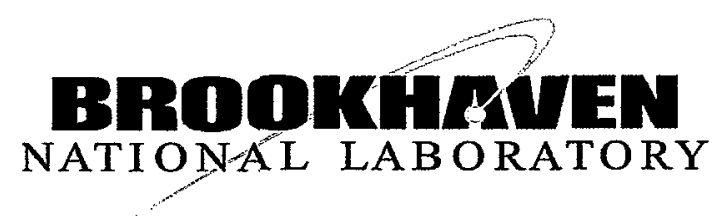

BNL-73454-2005-CP

\title{
Measurement of Transverse Echoes in RHIC
}

\author{
W. Fischer, T. Satogata, R. Tomas \\ Presented at the Particle Accelerator Conference (PAC'05) \\ Knoxville, Tennessee \\ May 16-20, 2005
}

\author{
Collider-Accelerator Department \\ Brookhaven National Laboratory \\ P.O. Box 5000 \\ Upton, NY 11973-5000 \\ www.bnl.gov \\ Managed by \\ Brookhaven Science Associates, LLC \\ for the United States Department of Energy under \\ Contract No. DE-AC02-98CH10886
}

This is a preprint of a paper intended for publication in a journal or proceedings. Since changes may be made before publication, this preprint is made available with the understanding that it will not be cited or reproduced without the permission of the author. 


\section{DISCLAIMER}

This report was prepared as an account of work sponsored by an agency of the United States Government. Neither the United States Government nor any agency thereof, nor any of their employees, nor any of their contractors, subcontractors, or their employees, makes any warranty, express or implied, or assumes any legal liability or responsibility for the accuracy, completeness, or any third party's use or the results of such use of any information, apparatus, product, or process disclosed, or represents that its use would not infringe privately owned rights. Reference herein to any specific commercial product, process, or service by trade name, trademark, manufacturer, or otherwise, does not necessarily constitute or imply its endorsement, recommendation, or favoring by the United States Government or any agency thereof or its contractors or subcontractors. The views and opinions of authors expressed herein do not necessarily state or reflect those of the United States Government or any agency thereof.

FOR UNCLASSIFIED, UNLIMITED STI PRODUCTS

Available electronically at:

OSTI:

http://www.osti.gov/bridge

Available for a processing fee to U.S. Department of Energy and its contractors, in paper from:

U.S. Department of Energy

Office of Scientific and Technical Information

P.O. Box 62

Oak Ridge, TN 37831

Phone: (865) 576-8401

Facsimile: (865) 576-5728

E-mail: reports@adonis.osti.gov

National Technical Information Service (NTIS):

Available for sale to the public from:

U.S. Department of Commerce

National Technical Information Service

5285 Port Royal Road

Springfield, VA 22131

Phone: (800) 553-6847

Facsimile: (703) 605-6900

Online ordering: http://www.ntis.gov/ordering.htm

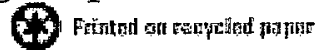




\title{
Measurement of Transverse Echoes in RHIC*
}

\author{
W. Fischer ${ }^{\dagger}$ and T. Satogata, Brookhaven National Lab, Upton, NY 11973, USA \\ R. Tomas, CELLS, Barcelona, Spain
}

\begin{abstract}
Beam echoes are a very sensitive method to measure diffusion, and longitudinal echo measurements were performed in a number of machines. In RHIC, for the first time, a transverse beam echo was observed after applying a dipole kick followed by a quadrupole kick. After application of the dipole kick, the dipole moment decohered completely due to lattice nonlinearities. When a quadrupole kick is applied at time $\tau$ after the dipole kick, the beam re-cohered at time $2 \tau$ thus showing an echo response. We describe the experimental setup and measurement results. In the measurements the dipole and quadrupole kick amplitudes, amplitude dependent tune shift, and the time between dipole and quadrupole kick were varied. In addition, measurements were taken with gold bunches of different intensities. These should exhibit different transverse diffusion rates due to intra-beam scattering.
\end{abstract}

\section{INTRODUCTION}

Echoes are well known in plasma physics, and were also introduced into beam physics [1]. Longitudinal beam echoes were observed in several machines, and used to measure diffusion rates [2-4]. The technique can also be used to measure transverse diffusion rates.

In the simplest case, a transverse echo is generated by a dipole kick followed by a quadrupole kick. The echo signal appears as a dipole moment long after the initial dipole oscillations have disappeared. We consider only this case, and follow in our notation Ref. [1].

With a dipole kick the particle distribution is displaced by several transverse rms beam sizes $\sigma$. If the particle tune is amplitude dependent the distribution filaments but information on the phase relations between the particles is still retained if the filamentation time is not too long. A quadrupole kick after time $\tau$ changes the distribution although it does not affect the dipole moment. After a time $\tau_{\text {echo }}=2 \tau$ a transient dipole moment appears, the echo signal, which can be observed with beam position monitors (BPMs). The process is illustrated in Ref. [5]. Fig. 1 shows a transverse echo measured in RHIC.

Echo measurements may be used to determine diffusion rates since any form of diffusion reduces the echo signal. The time of an echo measurement is considerably shorter than the time needed to observe the expansion of the beam size due to a diffusion process. Let $T_{0}$ be the revolution time, and $Q=\beta / f$ the normalized quadrupole strength, with $\beta$ the lattice function and $f$ the quadrupole focal length. We introduce the time $\tau_{0}=Q \tau$, and the decoherence time $\tau_{d}=T_{0} / 4 \pi \mu$, where $\mu$ is the betatron tune shift

\footnotetext{
${ }^{*}$ Work performed under the auspices of the US DOE.

†Wolfram.Fischer@bnl.gov
}

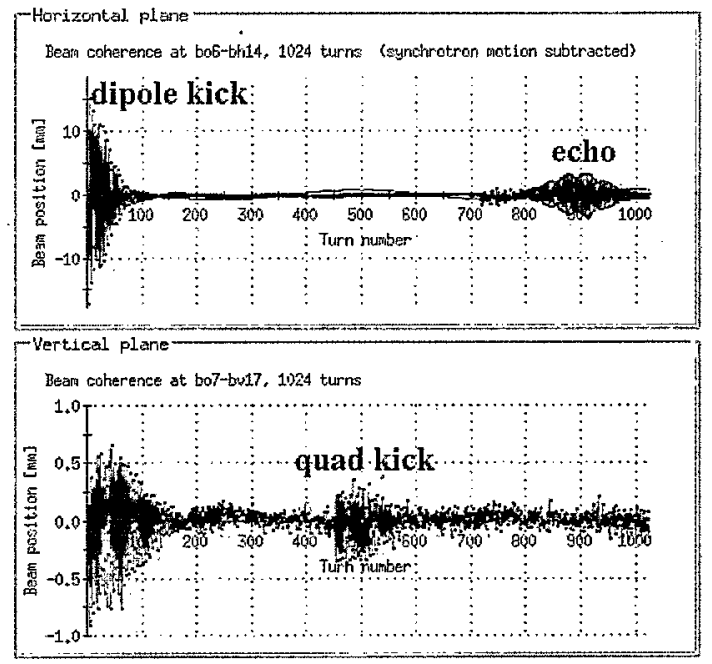

Figure 1: Beam oscillations seen in a horizontal and vertical BPM after injection. The beam is injected under a horizontal angle. After 450 turns a quadrupole kick is applied, faintly visible in the vertical plane. The transverse echo appears after 900 turns. Note the different scales for the horizontal and vertical plane.

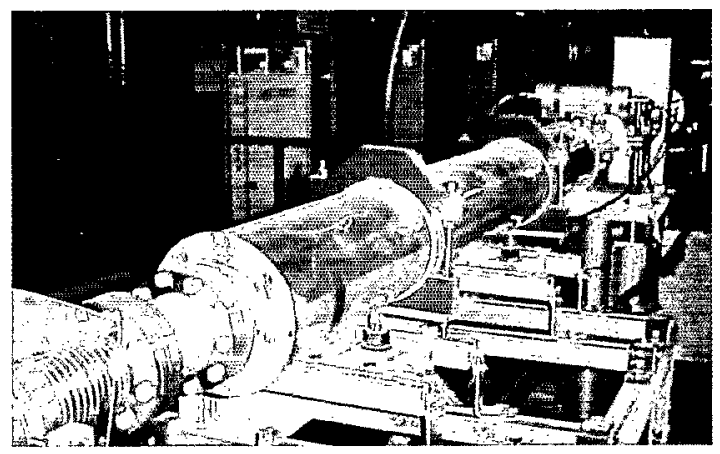

Figure 2: Pulsed quadrupole in the RHIC tunnel in a section common to both beams.

for particles at one rms beam size. For the case $\tau_{0} \ll \tau_{d}$ the echo amplitude $A_{\text {echo }}$ for a one-turn quadrupole kick wascalculated for one dimension in Ref. [1] as

$$
A_{e c h o}=\frac{\eta^{\max }}{a}=\frac{Q}{\tau_{d}} \frac{\tau}{1+8 D_{0} \mu^{2} \omega_{0}^{2} \tau^{3} / 3 \epsilon}
$$

where $\eta^{\max }$ is the maximum betatron amplitude in the echo signal, and $a$ the dipole kick. $\omega_{0}=2 \pi / T_{0}$, and $\epsilon$ is the rms of the unnormalized transverse particle distribution. $D_{0}$ is the diffusion coefficient. In the following we describe the measurement setup and report the measurement results. 
Table 1: Parameters of the pulsed quadrupole.

\begin{tabular}{lcc}
\hline parameter & unit & value \\
\hline mechanical length & $\mathrm{m}$ & 1.56 \\
inductance & $\mu \mathrm{H}$ & 105 \\
voltage & $\mathrm{kV}$ & 1.5 \\
current & $\mathrm{A}$ & 66 \\
focal length $f$ & $\mathrm{~m}$ & 407 \\
lattice $\beta_{x, y}$ & $\mathrm{~m}$ & 10.0 \\
normalized strength $Q$ &.. & 0.025 \\
rise and fall time & $\mu \mathrm{s} /$ turns & $12.8 / 1$ \\
\hline \hline
\end{tabular}

\section{SETUP}

The quadrupole kick is provided by an air-core quadrupole (see Fig. 2) that was originally used in the Tevatron's slow extraction system [6]. A pulsed power supply was built to obtain a one-turn kick [7]. The main parameters of the quadrupole are shown in Tab. 1.

All measurements were done at injection by observing the coherent transverse dipole oscillations with BPMs. For most measurements BPMs were used in a standard mode, in which 1024 turns are recorded. For a few measurements some BPMs were used in a special mode, in which up to one million turns can be recorded.

A dipole kick is created by injecting the beam under a horizontal angle, leading to dipole oscillations of about $10 \mathrm{~mm}$, or $3.5 \sigma$ (see Fig. 1). The dipole signal decoheres due to lattice nonlinearities in about 100 turns. The dominant lattice nonlinearities are created by octupoles in the arcs. After a number of turns, typically 450 , a quadrupole is pulsed. The quadrupole strength has a rise and fall time of one turn, and thus provides a one-turn kick. Since the orbit did not go exactly through the vertical center of the pulsed quadrupole, its effect can be seen as a weak signal in the vertical BPM. The echo is then visible later as a coherent dipole oscillation in the plane in which the dipole kick was applied. Typical parameters relevant to the measurements are shown in Tab. 2.

\section{MEASUREMENTS}

The echo amplitude was observed under variation of a number of parameters, and can be compared with expectations from Eq. (1). These were: the amplitude of the dipole kick $a$, the amplitude of the quadrupole kick $Q$, the detuning $\mu$, the timing of the quadrupole kick $\tau$, the horizontal tune $Q_{a z}$, and the bunch intensity $N_{b}$.

The echo amplitude $A_{\text {echo }}$ should be independent of the dipole kick. The dipole amplitude as varied between $5 \mathrm{~mm}$ and $11 \mathrm{~mm}$ and no significant change was observed. With even larger dipole kicks some beam is lost upon injection into the RHIC ring and the initial particle distribution is changed.

From Eq. (1) a linear dependence of the echo amplitude on the quadrupole kick strength is expected. Fig. 3 shows this scan. Up to almost the maximum quadrupole strength a linear dependence is observed. Close to the maximum strength a saturation effect becomes visible.
Table 2: Typical parameters for transverse echo measurement with Au beams in RHIC.

\begin{tabular}{lcc}
\hline \hline parameter & unit & value \\
\hline mass and charge number $A, Z$ & $\ldots$ & 197,79 \\
relativistic $\gamma$ & $\ldots$ & 10.52 \\
revolution time $T_{0}$ & $\mu \mathrm{s}$ & 12.8 \\
rms emittance, unnorm. $\epsilon$ & $\mathrm{mm} \cdot \mathrm{mrad}$ & 0.16 \\
detuning $\mu$ & $\ldots$ & 0.0014 \\
decoherence time $\tau_{d}$ & turns & 57 \\
dipole kick $a$ & $\mathrm{~mm} / \sigma$ & $10 / 3.5$ \\
quadrupole kick $Q$, time $\tau_{0}$ & $\ldots$, turns & $0.025,10$ \\
quadrupole kick time $\tau$ & turns. & 450 \\
bunch intensity $N_{b}$ & $10^{9}$ & $0.1-1.0$ \\
\hline \hline
\end{tabular}

The quadrupole kick time $\tau$ was varied between 0 and 500 turns. Only in the small range from 400 to 550 turns an echo was observed. In a later experiment with $\mathrm{Cu}$ beams, echoes could be observed from 100 to 1000 turns, with a maximum between 450 and 600 turns. In a few cases multiple echoes could be seen at times $2 \tau, 4 \tau$, and $6 \tau$. One example is shown in Fig. 5).

The detuning $\mu$ was changed with arc octupoles. A focusing and defocusing family at both dispersive and nondispersive locations exist in each sextant, with a total of 24 families with 132 magnets. All octupoles were set at the same strength. The detuning $\mu$ is calculated with a SixTrack [8] model of RHIC, and its value is consistent with the observed decoherence time (see Tab. 2). The scan is depicted in Fig. 4, along with calculation using Eq.(1) and three different values for $D_{0}$. With no additional lattice nonlinearities, no echoes can be observed. With increasing octupole strength, the echo amplitude $A_{e c h o}$ increases and reaches a maximum. The amplitude falls off with further strength increase since the memory time of the particles in the transverse distribution is reduced.

The measured curve in Fig. 4 cannot be well reproduced with Eq. (1). Of all the parameters in the equation, only the diffusion coefficient $D_{0}$ is unknown. All other parameters can be determined within $20 \%$ accuracy. We can offer a number of reasons for the discrepancy. First, with $\tau_{0}=$ 10 turns and $\tau_{d}=57$ turns the condition $\tau_{0} \ll \tau_{d}$ may be

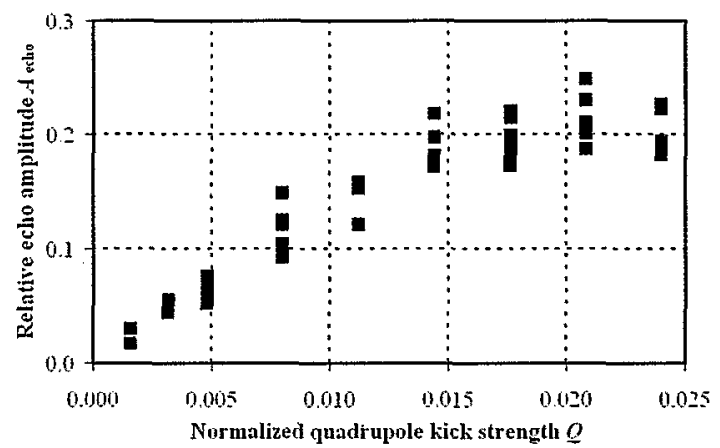

Figure 3: Relative echo amplitude $A_{\text {echo }}$ as a function of the quadrupole kick strength $Q$. 


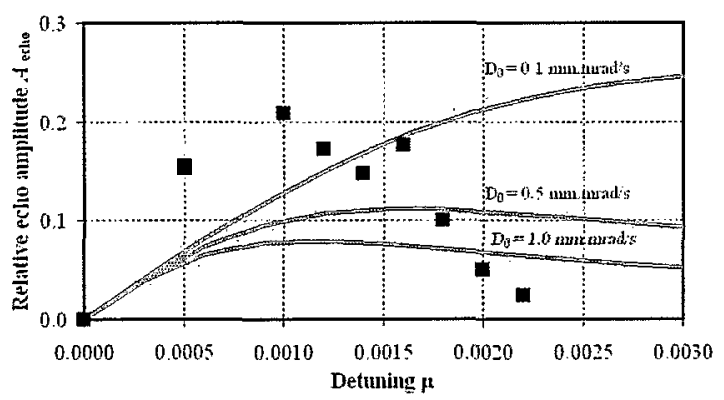

Figure 4: Measured relative echo amplitude $A_{\text {echo }}$ as a function of the detuning $\mu$ (blue squares) and calculated echo amplitude using Eq.(1) for three different diffusion coefficients (red lines).

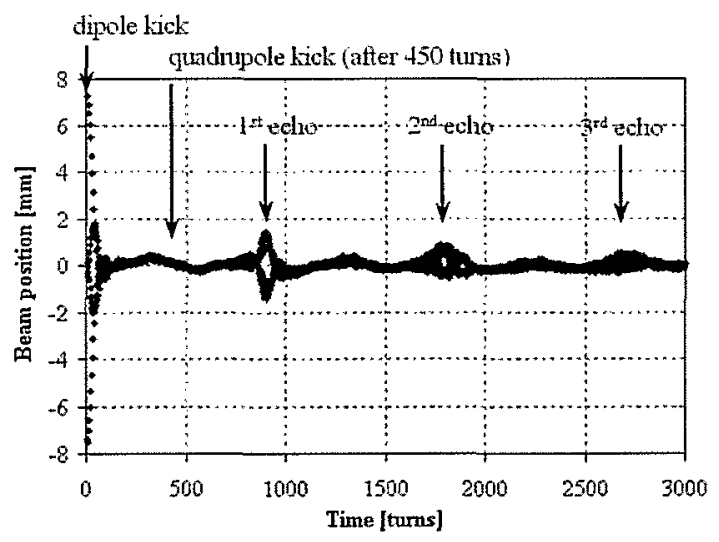

Figure 5: Observation of multiple echoes.

already sufficiently violated. Second, in the derivation of Eq. (1) a dipole kick much smaller than the beam size was assumed [9]. Third, only amplitude diffusion was taken into account while phase diffusion can also diminish the echo. Forth, the diffusion coefficient $D_{0}$ was assumed to be constant for the whole phase space. Even for rest gas scattering, the diffusion coefficient is proportional to the action [10], for many other processes it should grow even stronger with the betatron amplitude.

The dependence of the echo amplitude on the transverse diffusion rates was tested in 2 ways. First, the transverse tune was scanned. Close to a low order resonance, enhanced transverse diffusion may occur. No strong dependence of the echo amplitude on the horizontal tune was observed. Second, the bunch intensity was varied from $0.2 \times 10^{9}$ to $0.9 \times 10^{9}$, and the transverse diffusion rate changes due to intra-beam scattering [11]. In Fig. 6 the echo amplitude is shown as a function of the bunch intensity. From this the diffusion coefficient can be extracted in principle. Since Eq. (1) failed to reproduce the $\mu$ scan, we cannot use it to determine the diffusion coefficient. To obtain diffusion rates, one either needs to refine Eq. (1), or use a simulation to fit the diffusion rate. In 2005 data were also taken with $\mathrm{Cu}^{29+}$ beams, for which echoes were also observed. The analysis of those data is pending.

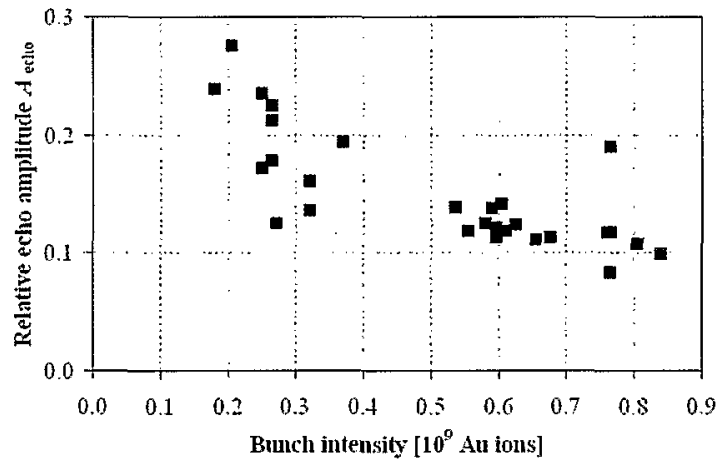

Figure 6: Relative echo amplitude $A_{e c h o}$ as a function of the bunch intensity.

\section{SUMMARY}

For the first time transverse echoes were measured in RHIC by applying a dipole kick, followed by a one-turn quadrupole kick. The echo amplitude was observed for changes in a number of parameters. In particular, the observed echo amplitude decreased with an increase in the bunch intensity, likely caused by an increase in the transverse diffusion rates due to intrabeam scattering. For lack of an adequate model, no diffusion coefficients could be obtained from these measurements yet.

\section{ACKNOWLEDGMENTS}

The authors are thankful for discussions and help to J. Adessi, O. Boine-Frankenheim, O. Brüning, J. Butler, A. Chao, O. Dressler, B. Nash, B. Parker, J. Piacentino, G. Stupakov, Y. Tan, S. Tepikian, C. Theisen, D. Trbojevic, D. Warburton, and W. Zhang.

\section{REFERENCES}

[1] G. Stupakov, "Echo", in "Handbook of accelerator physics and engineering" edited b A.W. Chao and M. Tigner, World Scientifi c (1999).

[2] L.K. Spentzouris, J.-F. Ostiguy and P.L. Colestock, "Direct measurement of diffusion rates in high energy synchrotrons using longitudinal beam echoes", PRL Vol. 76, No 4, pp. 620 (1996).

[3] O. Brüning et al., "Beam echos in the CERN SPS", PAC'97.

[4] J. Kewisch and M. Brennan, "Bunched beam echos in the AGS", EPAC'98.

[5] W. Fischer, B. Parker, and O. Brüning, "Transverse Echos in RHIC", proceedings of the US-LHC Collaboration Meeting on Accelerator Physics Experiments for Future Hadron Colliders, Upton, New York, BNL-5260l (2000).

[6] W. Fischer, A. Jain, and D. Trbojevic, "The AC quadrupole in RHIC". BNL RHIC/AP/164 (1999).

[7] O. Dressler, "Quadrupole Kicker for RHIC", BNL CA/AP/60 (2001).

[8] F. Schmidt, "SixTrack, User's Reference Manual", CERN/SL/94-56 (AP) (Update March 2000).

[9] G. Stupakov and A. Chao, "Effect of diffusion on bunched beam echo", PAC'97.

[10] M. Seidel, "Determination of diffusion rates in the proton beam halo of HERA", DESY HERA 93-04 (1993).

[11] W. Fischer et al., "Measurements of Intra-Beam Scattering Growth Times with Gold Beam below Transition in RHIC", PAC'01. 